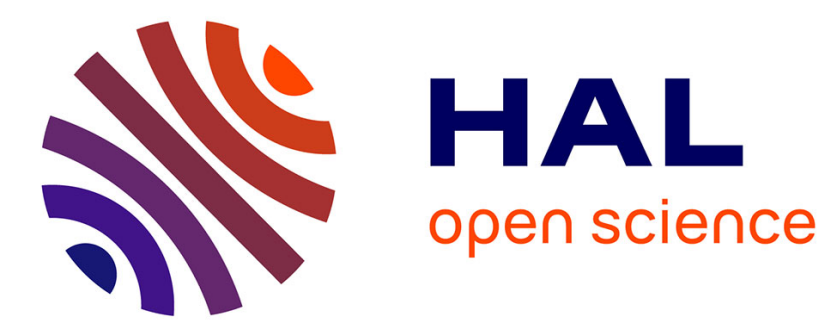

\title{
Les facteurs psychologiques qui ont un effet sur la réussite des étudiants
}

Franck Amadieu, André Tricot

\section{To cite this version:}

Franck Amadieu, André Tricot. Les facteurs psychologiques qui ont un effet sur la réussite des étudiants. Psicologia USP, 2015, Réussite et échec en langues de spécialité, XXIV (2), 10.4000/apliut.5155 . hal-01404959

\section{HAL Id: hal-01404959 \\ https://hal.science/hal-01404959}

Submitted on 29 Nov 2016

HAL is a multi-disciplinary open access archive for the deposit and dissemination of scientific research documents, whether they are published or not. The documents may come from teaching and research institutions in France or abroad, or from public or private research centers.
L'archive ouverte pluridisciplinaire $\mathbf{H A L}$, est destinée au dépôt et à la diffusion de documents scientifiques de niveau recherche, publiés ou non, émanant des établissements d'enseignement et de recherche français ou étrangers, des laboratoires publics ou privés. 


\title{
Recherche et pratiques \\ pédagogiques en langues \\ de spécialité
}

Cahiers de l'Apliut

Vol. XXXIV Nº 2 | 2015

Réussite et échec en langues de spécialité

Notes

\section{Les facteurs psychologiques qui ont un effet sur la réussite des étudiants}

\author{
Psychological factors which have an effect on student success
}

Franck Amadieu et André Tricot

p. pagination en cours

\section{Résumés}

Français English

Cette note de recherche aborde la question des étudiants qui apprennent mieux que les autres. En se basant sur des travaux de psychologie des apprentissages, elle traite des facteurs psychologiques qui contribuent à la réussite des étudiants dans leurs apprentissages, quelle que soit la discipline. Les travaux de recherche nous montrent que les étudiants qui sont en réussite et qui progressent le plus ont un niveau important de connaissances initiales dans le domaine en question. En outre, ils sont porteurs de motivations qui agissent sur leur engagement, leur persistance, et leur choix dans les tâches d'apprentissage.

Un étudiant qui a pour but de maîtriser le domaine qu'il étudie, qui a confiance en ses compétences et qui considère l'évaluation comme un moyen d'apprendre aura davantage de chance de réussir. Un étudiant qui cherche plutôt à être performant au regard des autres, sera plus fragile face à l'échec et aura des performances plus faibles sur le long terme.

Des recommandations pédagogiques sont alors proposées pour favoriser les motivations des étudiants. Ensuite, l'article distingue les stratégies d'étude sur la base de leur efficacité pour un apprentissage profond ${ }^{1}$ : les stratégies les plus efficaces mises en œuvre par les étudiants les plus performants sont les stratégies d'organisation, d'élaboration et de métacognition. Enfin, l'article discute les relations entre motivations et stratégies d'étude en montrant que la mise en œuvre de stratégies efficaces est coûteuse pour les étudiants et qu'il faut donc qu'elle soit soutenue par certains éléments motivationnels. 
The paper addresses the issue about learners who outperform the others. Referring to studies in educational psychology, it deals with psychological factors that participate in the learning success of students whatever the discipline is. The research works stress that students who succeed and progress the most have a high level of prior domain knowledge. Besides, they have motivations that support their engagement, their persistence, and their choices for their learning tasks.

Students who aim at mastering the studied domain, who believe in their competencies, and who consider exams as a way to learn, have a greater chance of success. Students who aim at being efficient in comparison to the others, are more affected by failure and perform less well in the long run.

Some teaching recommendations are given to favour students' motivations. Next, the paper distinguishes study strategies with regards to their efficiency for deep learning: the most efficient strategies conducted by the most successful students are organisation, elaboration and metacognitive strategies. Finally, the paper discusses the links between motivations and study strategies by showing that using efficient strategies is demanding for students and that their use has to be supported by motivations.

\section{Entrées d'index}

Mots-clés : apprentissage autorégulé, motivations, recommandation pédagogique, réussite, stratégies d'étude

Keywords : motivations, self-regulated learning, success, study strategies, teaching recommandation

\section{Texte intégral}

\section{Introduction}

Comprendre pourquoi certains étudiants réussissent leurs études alors que d'autres échouent constitue un enjeu important pour les universités et les enseignants qui y exercent. Cet enjeu suscite l'intérêt des chercheurs depuis une quarantaine d'années (par exemple, Tinto 1975). Ces chercheurs viennent de disciplines différentes et complémentaires, notamment la sociologie, les didactiques, les sciences du langage, l'économie ou la psychologie. Parfois ils essaient non seulement de comprendre mais aussi d'agir, en essayant de répondre à la question : comment favoriser la réussite des étudiants ? L'objectif de cette note de synthèse est de rendre compte de la contribution des chercheurs en psychologie à ce domaine de recherche. Quand cela est possible, nous rendrons compte des recherches conduites spécifiquement sur la réussite des étudiants en langues vivantes étrangères (LVE). En effet, la littérature générale sur la réussite des étudiants et la littérature spécialisée en enseignement apprentissage d'une LVE convergent très fortement pour les aspects que nous abordons, comme on le voit chez Rebecca Oxford et Jill Shearin (1994), Kata Csizér et Zoltán Dörnyei (2005), Zoltán Dörnyei (2009), Vera Busse et Catherine Walter (2013) et dans la synthèse générale de L. Quentin Dixon et ses collègues (2012).

$\mathrm{Au}$ cours de la première moitié du XXe siècle, les psychologues ont volontiers cherché des explications complexes, voire alambiquées (se focalisant plus souvent sur les élèves que sur les étudiants) : ceux qui réussissent sont plus intelligents ou plus doués que les autres, ils ont une personnalité qui favorise la réussite académique, un style cognitif ou d'apprentissage qui se prête bien aux études, etc. (voir la synthèse de Dörnyei 2009 pour la réussite en langues). Ces concepts du sens commun n'étant pas définis scientifiquement (pas plus que la catégorie « doué en langues »), les psychologues avaient bien du mal à sortir d'explications tautologiques. Au cours de la seconde moitié du XXe siècle, ils ont eu tendance à privilégier des explications plus «triviales »: les étudiants qui réussissent ont plus de connaissances dans la discipline que les autres 
(par exemple, plus de connaissances lexicales dans la langue étudiée) ; ces étudiants ont développé des motivations et des stratégies d'apprentissage mieux adaptées aux exigences de la formation. L'objectif de cet article est de présenter ces explications triviales.

L'article est organisé en cinq points. Le premier est consacré au niveau de connaissances des étudiants qui impacte directement leur réussite. Le second point traite des motivations des étudiants qui réussissent, ou, au moins, qui s'engagent dans leurs études. Le troisième point recense les pratiques pédagogiques qui peuvent contribuer à améliorer les motivations des étudiants. Le quatrième point abordera les attentes des étudiants par rapport aux enseignants. Le cinquième point exposera les grands types de stratégies d'étude en mettant en exergue ceux qui favorisent les apprentissages les plus profonds, c'est-à-dire des apprentissages qui permettent l'intégration en mémoire des connaissances en mobilisant des traitements de l'information importants, notamment sémantiques. Enfin, des liens existant entre motivations et stratégies d'étude sont discutés dans le dernier point.

\section{Les connaissances des étudiants qui réussissent}

Les connaissances antérieures des étudiants ont un effet majeur sur leur réussite. Ce phénomène, parfois connu sous le nom d' " effet Matthieu », a bien entendu été étudié par les sociologues et les économistes de l'éducation. Les psychologues ont aussi contribué à une meilleure compréhension de cet effet. En première approche, l'effet Matthieu peut sembler banal : les étudiants les plus avancés ont plus de chance de réussir à l'examen, car ils sont plus proches du but, du niveau à atteindre. Mais en regardant de plus près, on constate que ces étudiants ne font pas que réussir, ils progressent davantage. Récemment, notamment grâce aux travaux conduits au sein de la théorie de la charge cognitive (Sweller, Ayres et Kalyuga 2011; Diao et Sweller 2007 pour un exemple en LVE), on a compris que les apprenants qui ont plus de connaissances ont aussi plus de facilités pour réaliser les tâches qu'on leur propose, notamment quand celles-ci sont complexes et/ou exigeantes. Cette facilité à réaliser la tâche libère des ressources cognitives pour l'apprentissage lui-même, c'est-à-dire pour l'élaboration de nouvelles connaissances. Par exemple, une tâche de compréhension écrite peut être utilisée comme moyen pour un objectif d'acquisition de connaissances lexicales (ou autre, peu importe ici). Un étudiant qui a déjà beaucoup de connaissances sur la langue réussira mieux la tâche de compréhension écrite, et, conséquemment, aura plus de ressources cognitives pour acquérir de nouvelles connaissances lexicales (ou autre). Le manque de connaissances, tout comme une tâche trop exigeante, fonctionne comme une double peine : en pénalisant la réalisation de la tâche on pénalise l'apprentissage (Roussel et Tricot 2014).

\section{Les motivations des étudiants qui réussissent}

La motivation est un construit qui renvoie au but que poursuit une personne. La motivation est observable : une personne motivée s'investit dans la tâche censée lui permettre d'atteindre ce but et elle persévère quand cette tâche devient difficile. La motivation est influencée par différents facteurs psychologiques : valeur du but, attente de réussite, sentiment de contrôle sur la situation d'apprentissage et sentiment d'être compétent dans le domaine. On retrouve par exemple ces facteurs dans l'étude de 
Wendy McMillan (2010), consacrée aux étudiants les plus en réussite dans une faculté d'odontologie en Afrique du Sud. Ceux-ci répondaient à des questionnaires et à des entretiens et étaient observés dans certaines situations d'étude. Les résultats confirment les quatre points énumérés ci-après.

- Les étudiants qui réussissent accordent une valeur importante aux apprentissages académiques. Cette valeur renvoie à l'intérêt de l'étudiant pour le domaine étudié mais aussi à l'importance et à l'utilité accordées par l'étudiant aux thèmes abordés (Pintrich et Schrauben 1992).

- Les étudiants qui réussissent ont des buts de maîtrise : ils étudient dans le but de devenir par exemple dentistes avec une pratique sûre et efficace. On distingue classiquement les buts de performance, fondés sur la norme et la comparaison sociale, des buts de maîtrise du domaine et de plaisir (Decy et al 1991). Le but de maitrise impliquerait une évaluation auto-référencée de l'étudiant, organisée autour de la progression personnelle. Les étudiants poursuivant des buts de maitrise persistent davantage dans les tâches et sont moins vulnérables à l'échec, car l'échec est évalué et perçu comme un moyen de comprendre ses lacunes et donc va guider la mise en œuvre de nouvelles stratégies plus adaptées.

- Les étudiants qui réussissent ont un bon sentiment d'auto-efficacité : ils pensent qu'ils ont les compétences pour réaliser le type de tâche visé, ils ont confiance dans ces compétences (Zimmerman 2000). Les étudiants qui se sentent capables de conduire leur apprentissage vont davantage mettre en œuvre des stratégies d'étude adaptées. Ils prennent du plaisir dans certaines tâches qui peuvent être plus exigeantes mais qui présentent un certain niveau de défi.

- Enfin, ces étudiants font preuve de plus de persistance dans les tâches et ils cherchent davantage des solutions alternatives aux situations de problèmes étudiés. Les examens imposés et leur évaluation sont perçus par ces étudiants comme des étapes du processus d'apprentissage et non comme une sanction.

10 L'utilité est particulièrement importante dans l'apprentissage des LVE : dès que la langue apprise est perçue comme utile (l'étudiant est convaincu qu'il a besoin d'apprendre cette langue) ou objectivement utile (le contexte linguistique impose de pratiquer la langue), alors son apprentissage est beaucoup plus efficace. Les buts de maitrise et le sentiment d'auto-efficacité ont eux aussi un effet majeur dans l'apprentissage d'une LVE. Ces effets sont même dramatiquement importants : dans l'enseignement supérieur, s'il n'y a pas d'utilité perçue, la motivation intrinsèque et le sentiment d'auto-efficacité peuvent avoir tendance à baisser chez les étudiants (Busse et Walter 2013).

\section{Les pratiques pédagogiques qui favorisent les motivations pour apprendre}

Les recherches sur les pratiques pédagogiques montrent que si la motivation est bien une caractéristique des étudiants, elle est influencée par la façon dont on enseigne.

Il est alors important de montrer aux étudiants l'utilité des connaissances, notamment avec des mises en situation dans lesquelles les étudiants mesurent euxmêmes l'utilité de ces connaissances. Il est également important de montrer l'utilité des activités pédagogiques mises en place par l'enseignant. Il peut sembler évident pour un enseignant de faire réaliser telle ou telle activité à ses étudiants, mais ceux-ci n'ont pas toujours conscience de l'intérêt pédagogique de telle ou telle activité, même si c'est une pratique habituelle. Dans l'apprentissage d'une LVE, les approches qui favorisent les situations concrètes et proches de la réalité peuvent contribuer à motiver les étudiants. 
Cependant, ce n'est pas parce qu'une situation est effectivement proche de la réalité qu'elle va être perçue comme utile.

Proposer des tâches nouvelles, variées et avec un degré d'exigence raisonnable, permet de rompre avec une certaine monotonie des tâches habituelles. Varier les tâches permet de renouveler l'intérêt et l'engagement des étudiants, si et seulement si le niveau de défi reste raisonnable. Ce dernier aspect semble crucial en apprentissage d'une LVE. Quand on fait varier les tâches de manipulation d'une LVE, on augmente la motivation des étudiants, mais on aboutit parfois à un accident didactique, déjà mentionné plus haut : la difficulté de la tâche est telle que certains étudiants (les plus fragiles dans la LVE) ne parviennent pas à la réaliser (Roussel et al. 2008 ; Roussel 2011).

Favoriser une évaluation formative permet de maintenir un niveau de motivation élevé : un système d'évaluation centré sur le progrès et la maîtrise favorise le développement des buts de maîtrise chez les étudiants. L'évaluation doit pointer des compétences et leur maîtrise, aider l'étudiant à mesurer l'évolution de ses compétences, et donc son progrès. Les évaluations privées (non publiques) réduisent les comparaisons sociales entre étudiants. L'analyse approfondie des erreurs et de leurs causes peut amener les étudiants à considérer l'erreur de manière constructive. Dans les évaluations normatives l'erreur est généralement une situation à éviter et qui peut être source de stress pour certains étudiants.

Développer l'intérêt situationnel est un moyen de développer l'intérêt personnel : ce dernier renvoie à l'intérêt de l'étudiant pour une discipline ou un domaine sur une longue période. Cet intérêt est plutôt stable dans le temps et peu sensible à des effets de contexte. L'intérêt situationnel correspond à l'intérêt que l'étudiant porte à une situation particulière de cours. Cet intérêt est très sensible au contexte, et par conséquent reste fluctuant. Il est difficile d'avoir une action directe sur l'intérêt personnel, mais ce dernier peut évoluer sur la base d'un intérêt situationnel comme illustré dans la figure 1 ci-dessous.

\section{Figure 1 : Présentation des quatre étapes du modèle développement de l'intérêt} personnel de Suzanne Hidi et K. Ann Renninger (2006)

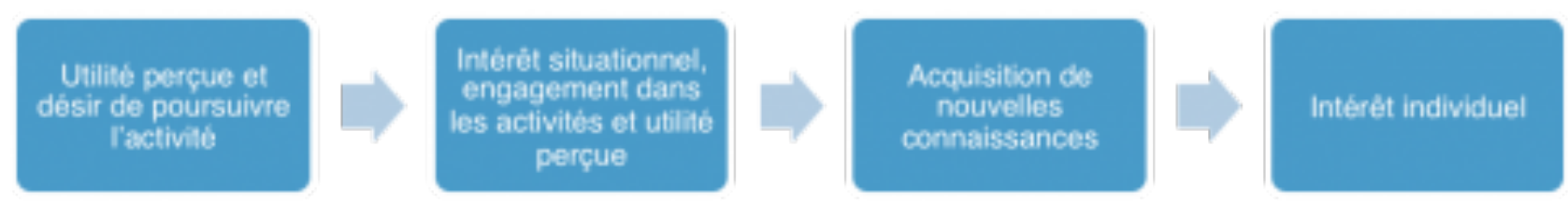

\section{Les attentes des étudiants par rapport aux enseignants}

En langues vivantes comme dans d'autres domaines, de nombreux travaux de recherche essaient de caractériser l'effet des enseignants et de leur travail sur la réussite des étudiants (voir la synthèse récente de Dixon et ses collègues, 2012). En psychologie, quelques recherches ont été consacrées à la perception des qualités des enseignants par les étudiants. Par exemple, Amit Khandelwal (2009) a conduit une étude auprès de 60 étudiantes en sciences humaines à l'Université de Delhi. Il était demandé aux étudiantes de se rappeler de situations dans lesquelles les enseignants étaient formidables dans leur enseignement et à l'inverse des situations où ils étaient médiocres. Des entretiens ont ensuite été conduits avec elles sur les différentes situations recensées. L'étude a abouti à un ensemble de dimensions. Nous présentons ici ces dimensions par ordre d'importance.

- Un rapport « harmonieux » avec l'enseignant, favorisé par une empathie, sympathie et indulgence de la part de l'enseignant. Comme dans toutes situations d'interaction entre êtres humains, l'attitude positive envers l'autre est un facteur de 
communication qui favorise un climat de confiance et de respect dans la situation de classe, et par conséquent, contribue à l'implication de l'autre dans les activités et les échanges.

- Préparation et conduite du cours. L'utilisation d'études de cas et d'anecdotes est positivement perçue par les étudiants, ainsi que les présentations qui permettent de relier les concepts à la vie de tous les jours. Ces attentes sont en cohérence avec la nécessité pour les étudiants de percevoir de l'utilité dans ce qu'ils apprennent. Les étudiants apprécient un savoir actualisé et d'actualité, les illustrations, les synthèses et les mises en lien entre les concepts. Ils apprécient un enseignant qui suscite la curiosité, qui utilise de la communication non-verbale, qui ne reste pas assis, qui ne lit pas son cours, qui est clair, qui favorise la participation.

- L'encouragement apporté par l'enseignant. Les étudiants apprécient un enseignant qui offre un soutien mais aussi montre de la confiance, qui croit en ses étudiants, qui ne réprimande pas, qui n'humilie pas, qui reconnait l'hétérogénéité du groupe. Les étudiants aspirent à être reconnus dans leur singularité et attendent de l'enseignant qu'il réponde à leurs besoins.

20 - Un enseignant « juste ». Pour les étudiants, un enseignant doit avoir un comportement juste et approprié aux circonstances ; il doit traiter de manière équitable les étudiants.

21 - Un enseignant disponible. Un enseignant qui passe du temps avec ses étudiants est apprécié. Les étudiants apprécient que les enseignants leur donne leur numéro de téléphone et adresse email, et qu'ils répondent rapidement aux emails et aux sollicitations.

\section{Les stratégies d'apprentissage}

L'enseignement des langues vivantes a été pionnier dans le domaine des stratégies d'apprentissage, leur accordant une grande importance dès le début des années 1990, et remplaçant avantageusement les travaux sur les styles d'apprentissage : alors que ces derniers seraient innés, intrinsèquement liés à l'individu, les stratégies d'apprentissage peuvent être apprises et enseignées. Comprendre quelles stratégies contribuent à un meilleur apprentissage et devenu depuis un enjeu majeur dans à peu près tous les domaines.

Classiquement, on distingue quatre grands types de stratégies d'apprentissage chez les étudiants. Elles ne renvoient pas toutes aux mêmes activités mentales, certaines étant plutôt de bas niveau alors que d'autres sont de haut niveau et par conséquent contribuent à un apprentissage plus profond (i.e. traitement important du sens). Le type de stratégie de bas niveau est de type “répétition". Il s'agit pour l'étudiant d'apprendre par cour, de recopier ou encore de relire son cours. Ce type de stratégies se caractérise donc par une activité cognitive qui fait peu appel aux connaissances antérieures de l'étudiant. Ce type de stratégie conduit généralement à des apprentissages de surface.

Les stratégies d'organisation consistent quant à elles à traiter en priorité les principales informations des contenus. Ces stratégies sont mises en œuvre lors d'activités dans lesquelles les étudiants résument le cours, font des fiches, structurent un plan du cours, etc. Elles sont donc de plus haut niveau que les stratégies de répétition, car elles contribuent à une activité cognitive de mise en relation des informations et d'organisation de celles-ci de manière à faciliter leur maintien et intégration en mémoire.

25 Un type de stratégie plus active encore concerne les stratégies d'élaboration. Ces stratégies consistent à faire des analogies avec d'autres situations étudiées, à relier les nouvelles informations à ses connaissances antérieures, à paraphraser le cours avec ses 
propres mots, à produire des liens entre les idées du cours et celles d'autres cours. En d'autres termes, ces stratégies concernent l'élaboration de nouvelles connaissances sur la base des connaissances initiales de l'étudiant. En élaborant des relations entre les nouvelles informations et leurs connaissances, mais aussi entre les nouvelles informations, les étudiants effectuent des traitements profonds de l'information et donc ont une meilleure intégration des informations en mémoire. Ces meilleures intégrations et appropriations des connaissances permettent un meilleur transfert de ces connaissances dans de nouvelles situations.

Enfin, les stratégies métacognitives sont typiques des stratégies de haut niveau. Elles contribuent aux activités de planification de l'activité d'apprentissage, aux autoévaluations, et à la régulation de leur apprentissage. C'est parce que les étudiants sont capables d'évaluer la pertinence de leurs comportements d'apprentissage qu'ils peuvent les modifier. Si un comportement ne permet pas d'atteindre efficacement ou rapidement les objectifs, alors il faudra le modifier, voir le remplacer par un autre. On comprend pourquoi ces stratégies sont essentielles dans les apprentissages autorégulés.

Jeffrey Karpicke, Andrew Butler et Henry Roediger (2009) se sont intéressés aux stratégies que les étudiants de leur université aux États-Unis mettaient en œuvre. Ils ont demandé à 177 étudiants de lister les stratégies qu'ils utilisaient. Les résultats obtenus sont présentés dans le tableau 1.

Tableau 1 : Liste des stratégies d'étude rapportées par les étudiants par fréquence d'apparition dans les listes des étudiants

\begin{tabular}{|lc|}
\hline Stratégies rapportées par les étudiants & $\%$ \\
\hline Relecture des notes et documents & 83.6 \\
\hline Faire des exercices & 42.9 \\
\hline Faire des fiches & 40.1 \\
\hline Réécrire les notes & 29.9 \\
\hline Étudier en groupe & 26.5 \\
\hline Apprendre par cœur & 18.6 \\
\hline Moyens mnémotechniques & 13.5 \\
\hline Faire des plans & 12.9 \\
\hline Rappel (auto-évaluation) & 10.7 \\
\hline Souligner (notes ou documents) & 6.2 \\
\hline Penser à des exemples de la vie de tous les jours & 4.5 \\
\hline
\end{tabular}

Les stratégies de surface (les stratégies de bas niveau telle que la " relecture ») sont majoritaires. D’autres stratégies du même ordre ( « apprendre par cœur » et « moyens mnémotechniques ») sont aussi utilisées. Les stratégies plus élaborées (i.e. stratégies de haut niveau), sont représentées par " faire des fiches », " réécrire les notes », " faire des plans » et « souligner ». Ensuite, les stratégies d'élaboration sont représentées par « faire des exercices » (si l'on considère que l'exercice implique de l'élaboration et non simplement une routinisation de savoir-faire), " étudier en groupe » et «penser à des exemples de la vie de tous les jours ». Elles sont assez peu utilisées en comparaison aux autres types de stratégies. Ces étudiants utilisent donc peu des stratégies de haut niveau. 
niveau, sont peu utilisées. Elles consistent pour l'étudiant à s'auto-évaluer en essayant de rappeler les principaux éléments du cours ou en essayant de répondre à une évaluation sous forme de questions, par exemple. Ce type de stratégie, qui ne représente que $10 \%$ des stratégies rapportées par les étudiants, est pourtant utile pour l'apprentissage. Le fait de s'auto-évaluer permet certes de mesurer l'écart entre ce que l'on sait et ce que l'on devrait savoir, mais aussi de récupérer en mémoire des informations et donc de fournir un travail d'élaboration du savoir. Cette activité de récupération en mémoire permet un meilleur ancrage des connaissances en mémoire. En d'autres termes, pour apprendre il faut se tester, d'où le nom donné à cet effet, «l'effet testing ».

30 Peu d'étudiants semblent donc se tester pour apprendre, et lorsque l'on demande à des étudiants pour quelles raisons ils se testent lorsqu'ils révisent un cours, $68 \%$ déclarent le faire pour évaluer ce qu'ils ont compris (Kornell et Bjork 2007). Seulement 18\% des étudiants déclarent le faire parce qu'ils apprennent mieux ainsi qu'en relisant. Les étudiants ne sont pas conscients des bénéfices de l'effet testing. Ce problème, qui relève d'un problème de connaissances métacognitives, vient du fait que les étudiants n'ont pas forcément une bonne représentation des stratégies d'apprentissage qui sont efficaces ou non.

Wendy McMillan (2010) montre que les étudiants qui réussissent font de nombreux résumés et de nombreuses paraphrases des contenus de cours. Ils cherchent à identifier les idées principales du cours et organisent ces idées dans des tables des matières ou autres systèmes d'organisation d'ensemble. Ils utilisent donc beaucoup de stratégies d'organisation. Leurs prises de notes sont synthétiques, ils relisent leurs notes pour les ajuster et les étudier. Ces étudiants font des plannings d'étude et donc anticipent et organisent leurs activités d'apprentissage sur une période déterminée. De plus, ils rédigent des questions à poser à l'enseignant ou à rechercher soi-même, et ils cherchent à connaître les modalités et exigences des évaluations.

Dans l'apprentissage d'une LVE, les aspects stratégiques jouent un rôle très important. Cette importance est reconnue depuis le début des années 1990, avec les ouvrages de Michael O’Malley et Anna Chamot (1990), Rebecca Oxford (1990) et Anita Wenden (1991). À la suite de ces auteurs, on peut par exemple distinguer entre :

- $\quad$ les stratégies cognitives : savoir agir sur le support d'apprentissage ;

- les stratégies métacognitives : savoir planifier, gérer, organiser et évaluer son propre apprentissage ;

- $\quad$ les stratégies sociales : savoir initier des interactions et des coopérations ;

- les stratégies affectives : savoir prendre le contrôle de ses émotions en situation d'apprentissage d'une LVE.

33 Plusieurs études montrent que ces stratégies, tout comme l'auto-évaluation, sont enseignables en LVE. Des scénarios d'enseignement de ces stratégies ont été élaborés et mis à l'épreuve (voir, par exemple, Macaro 2001). Aujourd'hui encore donc, les travaux sur les stratégies d'apprentissage en LVE semblent particulièrement riches et innovants.

\section{Les liens entre motivations et stratégies d'étude}

Nous avons abordé jusqu'ici deux grands types de facteurs de la réussite des étudiants que sont les motivations et les stratégies d'étude, mais nous n'avons pas encore abordé les relations qu'entretiennent ces deux facteurs. La question, finalement, est de savoir si les stratégies d'étude mises en œuvre par les étudiants dépendent de 
leurs motivations. Pour y répondre, nous présentons deux ensembles de travaux empiriques qui ont examiné les relations entre le type de motivations et de stratégies d'étude des étudiants.

De nombreux travaux ont été menés sur la question des facteurs motivationnels qui amènent les étudiants à mettre en œuvre des stratégies d'étude de haut niveau, à savoir des stratégies d'organisation et d'élaboration. Un grand nombre de recherches sur cette question a été mené par Paul Pintrich et ses collaborateurs (Pintrich et De Groot 1990 ; Pintrich et García 1993). Dans l'ensemble, ces travaux nous montrent que les performances obtenues aux examens sont prédites par l'utilisation de stratégies d'élaboration et par des activités d'autorégulation lors des apprentissages dues à des compétences métacognitives. Ces stratégies à l'origine des performances aux examens sont en fait elle-même prédites par des facteurs d'ordre motivationnel tels que le sentiment d'auto-efficacité et valeur accordée à la tâche. Ainsi, pour mettre en œuvre des stratégies de haut niveau, il faut pouvoir s'engager dans la tâche, ce qui nécessite de se sentir capable de réaliser les apprentissages et de considérer que ce que l'on étudie est important. Ces travaux indiquent que les motivations intrinsèques, comme les buts de maîtrise, favorisent la mise œuvre de stratégies de haut niveau. À l'inverse, les étudiants qui ont plutôt des buts de performance mettent davantage en œuvre des stratégies de surface (Darnon et Butera 2005 ; Nolen 1988).

36 Une étude d'Alberto Valentin et al. (2013) illustre une nouvelle fois les relations entre les aspects motivationnels et les stratégies d'étude des étudiants. Le point original de l'étude est que l'auteur s'est intéressé à l'usage d'une plateforme pédagogique en ligne de type Moodle. Tout d'abord, l'étude montre qu'il n'y avait pas véritablement de liens entre l'usage de la plateforme et les performances aux examens. En revanche, l'usage de la plateforme était plus important chez les étudiants qui avaient des motivations intrinsèques, donc plutôt des buts de maîtrise, qui accordaient une valeur importante à la tâche et qui employaient des stratégies d'élaboration. En d'autres termes, une plateforme numérique d'apprentissage, non obligatoire, est principalement utilisée par des étudiants en réussite. En fait, ce type de plateforme exige une autorégulation de l'apprentissage, et donc des compétences d'étude et des motivations plutôt intrinsèques chez les étudiants. Enfin l'étude a confirmé que les étudiants qui avaient des motivations intrinsèques et qui accordaient une valeur importante à la tâche d'étude étaient ceux qui avaient le plus recours à des stratégies d'élaboration.

\section{Conclusion}

Dans cette note, nous n'avons bien sûr pas pu étudier tous les facteurs psychologiques qui ont un effet sur la réussite des étudiants. En revanche, nous avons pointé des éléments qui pourront aider chaque enseignant à comprendre certaines différences entre ses étudiants et, par là, construire des activités pédagogiques qui soutiendront encore mieux l'apprentissage. Bien entendu, d'autres facteurs que ceux traités ici peuvent expliquer les différences d'apprentissage entre nos étudiants, par exemple des facteurs de type socio-culturel ou encore émotionnel.

Pour apprendre sur la durée et pour espérer pouvoir réinvestir ses connaissances, il est nécessaire de mobiliser ses connaissances et de mettre en œuvre des stratégies de haut niveau lors de son apprentissage. Les étudiants doivent organiser l'information, élaborer et réguler leur apprentissage. Nous exigeons souvent de nos étudiants des apprentissages autorégulés qui nécessitent de l'adaptation, de l'autonomie et de l'engagement. Cet engagement nécessaire à la mise en œuvre de stratégies d'étude de haut niveau sera permis par certaines caractéristiques motivationnels des étudiants. La poursuite d'un but de maîtrise, le sentiment d'auto-efficacité et la valeur accordée aux tâches et au domaine sont des facteurs importants qui favoriseront le recours à des 
stratégies de haut niveau. Nous avons également pu voir que les étudiants n'étaient pas toujours conscients des stratégies qui étaient les plus adaptées et les plus efficaces pour leur apprentissage. Nous pouvons contribuer à améliorer les motivations de nos étudiants dans nos enseignements, mais aussi à les accompagner dans l'usage des bonnes stratégies à mettre en œuvre selon les situations et les exigences pédagogiques (ex. degré d'autonomie exigé dans les activités pédagogiques).

Dans l'enseignement et l'apprentissage d'une LVE, les études empiriques et théoriques consacrées à ces aspects sont relativement peu nombreuses. Mais, comme nous l'avons souligné dans cet article, il y a une très forte convergence entre les résultats de ces recherches spécialisées et celles, plus générales, que nous avons présentées. Il est donc possible de considérer les travaux que nous venons de présenter comme des sources d'inspiration possibles pour la conception et la mise en œuvre d'actions pédagogiques favorisant l'engagement et la réussite des étudiants.

\section{Bibliographie}

Busse, V. \& C. Walter. 2013. « Foreign language learning motivation in higher education: A longitudinal study of motivational changes and their causes. » The Modern Language Journal, 97(2), 435-456.

DOI : $10.1111 / \mathrm{j} \cdot 1540-4781.2013 .12004 \cdot \mathrm{x}$

Csizér, K. \& Z. Dörnyei. 2005. « Language learners' motivational profiles and their motivated learning behavior. » Language Learning, 55, 613-659.

DOI : 10.1111/j.0023-8333.2005.00319.x

Darnon, B. \& F. Butera. 2005. "Buts d'accomplissement, stratégies d'étude, et motivation intrinsèque : présentation d'un domaine de recherche et validation française de l'échelle d'Elliot et McGregor (2001) ». L’Année Psychologique, 105, 105-131.

Deci, E., L. R. J. Vallerland, L. G, Pelletier \& R. M. Ryan. 1991. « Motivation in education: the self-determination perspective. » The Educational Psychologist, 26, 325-346.

Diao, Y. \& J. Sweller, 2007. « Redundancy in foreign language reading comprehension instruction: Concurrent written and spoken presentations. » Learning and Instruction, 17(1), 78-88.

DOI : 10.1016/j.learninstruc.2006.11.007

Dixon, L. Q., J. Zhao, J., Shin \& al. 2012. " What we know about second language acquisition a synthesis from four perspectives. » Review of Educational Research, 82(1), 5-60.

DOI : $10.3102 / 0034654311433587$

Dörnyei, Z. 2009. The Psychology of Second Language Acquisition. Oxford: Oxford University Press.

Greene, J. A. \& R. Azevedo. 2007. « A theoretical review of Winne and Hadwin's model of selfregulated learning: New perspectives and directions. » Review of Educational Research 77(3), 334-372.

DOI : $10.3102 / 003465430303953$

Hidi, S. \& K. A. Renninger. 2006. "The four-phase model of interest development. » Educational Psychologist, 41, 111-127.

DOI : 10.1207/s15326985ep4102_4

Karpicke, J. D., A. C. Butler \& H. L. Roedige. 2009. " Metacognitive strategies in student learning: Do students practice retrieval when they study on their own? » Memory, 17, 471-479.

DOI : $10.1080 / 09658210802647009$

Khandelwal, K. A. 2009. "Effective teaching behaviors in the college classroom: A critical incident technique from students' perspective. "International Journal of Teaching and Learning in Higher Education, 21(3), 299-309.

Kornell, N. \& R. A. Bjork,. 2007. "The promise and perils of self-regulated study. » Psychonomic Bulletin \& Review, 14, 219-224.

DOI : $10.3758 /$ BFo3194055

Macaro, E. 2001. Learning Strategies in Foreign and Second Language Classrooms. London: Continuum.

McMillan, W. J. 2010. "Your thrust is to understand - how academically successful students learn. » Teaching in Higher Education, 15(1), 1-13. 
DOI : $10.1080 / 13562510903488105$

Nolen, S. B. 1988. "Reasons for studying: Motivational orientations and study strategies. » Cognition and Instruction, 5, 269-287.

DOI : 10.1207/s1532690xcio504_2

O'malley, J. M. \& A. U. Chamot. 1990. Learning Strategies in Second Language Acquisition. Cambridge University Press.

DOI : $10.1017 / \mathrm{CBO} 9781139524490$

Oxford, R.L. 1990. Language Learning Strategies: What Every Teacher Should Know. Boston: Heinle \& Heinle.

Oxford, R. \& J. Shearin. 1994. " Language learning motivation: Expanding the theoretical framework. » The Modern Language Journal, 78(1), 12-28.

DOI : 10.1111/j.1540-4781.1994.tbo2011.x

Pintrich, P. R. \& E.V. De Groot. 1990. « Motivational and self-regulated learning component of classroom academic performance. » Journal of Educational Psychology, 82(1), 33-40.

DOI : 10.1037/0022-0663.82.1.33

Pintrich, P. \& B. Schrauben. 1992. "Students' motivational beliefs and their cognitive engagement in classroom academic tasks. »In D.H. Schunk and J.L. Meece (Eds.) Student Perceptions in the Classroom, 149-175. Hillsdale, NJ: Erlbaum.

Pintrich, P. \& T. García. 1993. "Intraindividual diffrences in students' motivation and selfregulated learning. » German. Journal of Educational Psychology, 7(3), 99-107.

Roussel, S. 2011. «A computer assisted method to track listening strategies in second language learning. » ReCALL, 23(2), 98-116.

DOI : $10.1017 /$ So958344011000036

Roussel, S., A. Rieussec, J-L. Nespoulous \& A. Tricot. 2008. « Des baladeurs MP3 en classe d'Allemand. L'effet de l'autorégulation matérielle de l'écoute sur la compréhension auditive en langue seconde. » Alsic, 11(2).

DOI : $10.4000 /$ alsic.413

Roussel, S., \& A. Tricot, A. 2014. « Le numérique en classe : émancipation ou double peine ? ». In S. Brunel (dir.). De la didactique des usages numériques. Éditions universitaires europeennes : 119-140.

Sweller, J., P. Ayres \& S. Kalyuga. 2011. Cognitive Load Theory. New York: Springer.

Tinto, V. 1975. « Dropout from higher education: A theoretical synthesis of recent research. » Review of Educational Research, 45, 89-125.

DOI : 10.3102/00346543045001089

Valentín, A., P. M. Mateos, M. González-Tablas \& al. 2013. " Motivation and learning strategies in the use of ICTs among university students. » Computers \& Education, 61, 52-58.

DOI : 10.1016/j.compedu.2012.09.008

Wenden, A. (1991). Learner Strategies for Learner Autonomy. Hemel Hempstead: Prentice Hall.

Zimmerman, B. J. 2000. "Self-efficacy: An essential motive to learn. » Contemporary Educational Psychology, 25(1), 82-91.

DOI : $10.1006 /$ ceps.1999.1016

Zimmerman, B. J. 2008. « Investigating self-regulation and motivation: historical background, methodological developments, and future prospects. " American Educational Research Journal, 45(1), 66-83.

DOI : 10.3102/0002831207312909

\section{Notes}

1 Un apprentissage profond renvoie à un apprentissage signifiant qui implique la compréhension du sens des contenus d'un cours, tandis qu'un apprentissage en surface renvoie généralement à un apprentissage par répétition et à la mémorisation de textes impliquant de traitement du sens.

\section{Table des illustrations}


Titre de l'intérêt personnel de Suzanne Hidi et K. Ann Renninger (2006)

URL http://apliut.revues.org/docannexe/image/5155/img-1.png

Fichier image/png, 26k

\section{Pour citer cet article}

Référence papier

Franck Amadieu et André Tricot, « Les facteurs psychologiques qui ont un effet sur la réussite des étudiants ", Recherche et pratiques pédagogiques en langues de spécialité, Vol. XXXIV N ${ }^{\circ} 2$ | 2015, pagination en cours.

Référence électronique

Franck Amadieu et André Tricot, « Les facteurs psychologiques qui ont un effet sur la réussite des étudiants ", Recherche et pratiques pédagogiques en langues de spécialité [En ligne],

Vol. XXXIV N ${ }^{\circ} 2$ | 2015, mis en ligne le 15 juin 2015, consulté le 29 novembre 2016. URL :

http://apliut.revues.org/5155 ; DOI : 10.4000/apliut.5155

\section{Auteurs}

\section{Franck Amadieu}

Franck Amadieu est enseignant-chercheur en psychologie cognitive à l'Université Toulouse Jean Jaurès, membre du laboratoire CLLE-LTC. Ces travaux de recherche portent principalement sur les apprentissages des étudiants avec des technologies multimédia. II s'intéresse plus particulièrement aux stratégies d'apprentissages et d'interaction avec les supports multimédia afin de comprendre quels types de stratégies sont efficaces pour mémoriser et comprendre.

amadieu@univ-tlse2.fr

\section{André Tricot}

André Tricot est Professeur des universités en psychologie à l'Université Toulouse - Jean Jaurès, membre du laboratoire CLLE-LTC. Son travail de recherche concerne les apprentissages et la recherche d'information avec des documents numériques, selon une approche cognitive et ergonomique. Il s'intéresse aussi à la théorie de la charge cognitive et à l'ergonomie des IHM.

andre.tricot@univ-tlse2.fr

\section{Droits d'auteur}

Association des Professeurs de Langues des Instituts Universitaires de Technologie 